\title{
Quantitative genetic analysis of natural variation in body size in Drosophila melanogaster
}

\author{
J Gockel, SJW Robinson, WJ Kennington, DB Goldstein and L Partridge \\ Department of Biology, University College London, London, UK
}

Latitudinal, genetic variation in body size is a commonly observed phenomenon in many invertebrate species and is shaped by natural selection. In this study, we use a chromosome substitution and a quantitative trait locus (QTL) mapping approach to identify chromosomes and genomic regions associated with adaptive variation in body size in natural populations of Drosophila melanogaster from the extreme ends of clines in South America and Australia. Chromosome substitution revealed the largest effects on chromosome three in both continents, and minor effects on the $X$ and second chromosome. Similarly, QTL analysis of the Australian cline identified QTL with largest effects on the third chromosome, with smaller effects on the second. How- ever, no QTL were found on the X chromosome. We also compared the coincidence of locations of QTL with the locations of five microsatellite loci previously shown to vary clinally in Australia. Permutation tests using both the sum of the LOD scores and the sum distance to nearest QTL peak revealed there were no significant associations between locations of clinal markers and QTL's. The lack of significance may, in part, be due to broad QTL peaks identified in this study. Future studies using higher resolution QTL maps should reveal whether the degree of clinality in microsatellite allele frequencies can be used to identify QTL in traits that vary along an environmental gradient.

Heredity (2002) 89, 145-153. doi:10.1038/sj.hdy.6800121

Keywords: QTL mapping; Drosophila melanogaster, body size; wing area; natural variation; cline

\section{Introduction}

Morphological traits usually show continuous, quantitative variation in natural populations. For many such traits an underlying genetic basis has been demonstrated. Recently, many attempts have been made to identify the genetic loci underlying variation in several quantitative traits. The identification of such loci would be critical in an evaluation of models aiming to determine how genetic variation for adaptive quantitative traits is maintained in natural populations and whether the loci at which variation occurs within a population are the same as those that cause divergence between populations and species (Mackay, 2001).

The genetic architecture of quantitative traits has been examined by analysing natural variation among or within species and, in the majority of cases, variation among inbred laboratory strains that sometimes were artificially selected for trait variation. In particular, abdominal and sternopleural bristle number variation has been the focus of many such analyses. As an ideal model trait, it has been used for over 50 years to estimate fundamental quantitative genetic parameters and many candidate genes associated with trait variation have been characterized genetically (Long et al, 1995; Gurganus et al, 1999; Nuzhdin et al, 1999). Studies based on laboratory

Correspondence: L Partridge, Department of Biology, University College London, Darwin Building, Gower Street, London WC1E 6BT, UK. E-mail: l.partridge@ucl.ac.uk

Received 30 October 2001; accepted 28 April 2002 populations have further looked at other morphological and life history traits such as wing shape (Weber et al, 1999; Zimmerman et al, 2000) and longevity (Vieira et al, 2000; Leips and Mackay, 2000).

Fewer studies have aimed at detecting quantitative trait locus (QTL) of traits that vary between species like sexually selected morphological or hybrid incompatibility traits (Liu et al, 1996; Macdonald and Goldstein, 1999; Zeng et al, 2000). Likewise, only a few studies have investigated QTL for naturally occurring phenotypic variation within species. For instance, Laurie et al (1991) analysed associations between polymorphisms within the Adh gene and variation in ADH protein level for a number of chromosomes derived from natural populations. Other studies have mainly focussed on the allelic effects of candidate loci on bristle number variation (Lyman and Mackay, 1998; Lyman et al, 1999; Long et al, 2000). Identification of the precise DNA polymorphisms or quantitative trait nucleotides (QTN) that contribute to continuous variation are of great interest, as they will provide insight into the genetic nature of adaptation. Comparing the results of studies of quantitative traits at the within and among-species level will allow an evaluation of whether traits that diverge during speciation arise out of the standing variation within species. Furthermore, analysis of naturally occurring variation may reflect the genetic architecture underlying this trait under natural selection more accurately than variation based on artificial selection in the laboratory (Harshman and Hoffmann, 2000).

The causes of continuous variation of body size along latitudinal gradients have long been of major interest to evolutionary biologists. Among ectotherms, these studies 
have mainly focussed on Drosophila melanogaster for which clinal body size variation has been demonstrated on many different continents (Imasheva et al, 1994; James et al, 1995, 1997; vant Land et al, 1999). This repeatability of an increase in body size with latitude strongly suggests that variation of this trait has been shaped by natural selection. D. melanogaster has only recently colonized different regions around the world. It has been demonstrated, however, that the rate of evolution of body size clines in Drosophila can be very fast on a continental scale (Huey et al, 2000). Consequently, it would be interesting to identify the genes that have contributed to adaptation under natural conditions to understand the evolution of trait variation along environmental gradients. So far, QTL mapping has been restricted to a small number of model organisms for which a set of molecular mapping tools is available. Furthermore, the mapping procedure itself is costly and time consuming as laboratory maintenance and repeated crossing is necessary. It is therefore of great interest to develop methods that allow localization of QTL in a more efficient way that can also be applied to species for which conventional mapping approaches are not suited. Organisms that show clinal variation for a quantitative trait along an environmental gradient may provide an ideal scenario to analyze marker trait associations by testing for clinal allele frequencies of otherwise neutral molecular markers.

In this study we tested for chromosomal and QTL effects on natural variation in body size, measured as wing area, in D. melanogaster. To obtain an estimate of the relative contribution of each of the three major chromosomes to body size variation, a chromosome substitution procedure was employed in flies from latitudinal transects in Australia and South America. Clinal variation in body size in these flies has been described previously (James et al, 1995; vant Land et al, 1999). The presence of the Australian cline was reconfirmed in a later study (Gockel et al, 2001). Comparison of the chromosomal effects in two different clines further allowed us to determine whether the chromosomal basis of these two clines is the same. In both continents major effects have been found on the third chromosome and minor but still statistically significant effects on the $X$ and second chromosome.

These effects have been further analysed in a genomewide screen for QTL in the Australian cline. QTL mapping was carried out for crosses of inbred lines that were set up from populations at the extreme ends of the clinal distribution to obtain maximum phenotypic difference between the parental lines. We employed a genetic map using microsatellite markers with an average spacing of $7.1 \mathrm{cM}$ across the three major chromosomes of the Drosophila genome. Using composite interval mapping, we show that the largest part of genetic variation is explained by QTL found on the third chromosome, confirming the results of the chromosome substitution. No QTL were detected on the $X$ and only minor effects on the second chromosome. To obtain indirect estimates of QTL locations in populations along the Australian body size cline, microsatellite markers that previously have been shown to express different levels of clinality (Gockel et al, 2001) were tested for their positions underneath the QTL probability curve. Clinal markers, ie markers for which latitude explained a significant proportion of the total variation, showed a greater tendency to be located underneath a QTL peak, but this trend was not significant.

\section{Materials and methods}

\section{Chromosome substitution}

The populations used have been described elsewhere (Azevedo et al, 1996; Gilchrist and Partridge, 1999; vant Land et al, 1999; Zwaan et al, 2000). Briefly, the South American populations were collected in 1995 from Guayaquil (Equador, 2.22 ${ }^{\circ}$ ) and Puerto Montt (Chile, $\left.41.50^{\circ} \mathrm{S}\right)$. The Australian populations were collected in January 1997, from sites at Cygnet (Tasmania, $43.15^{\circ} \mathrm{S}$ ) and Innisfail (Queensland, $17.30^{\circ} \mathrm{S}$ ). All populations have been maintained since collection in bottle culture at $25^{\circ} \mathrm{C}$. Over the course of five generations, individual chromosomes from low latitude (Small) lines (SSS) were inserted one at a time into the high latitude (Big) background (BBB) and vice-versa to produce all six possible combinations of substitution lines (SBB, BSB, BBS, BSS, SBS, $\mathrm{SSB})$. Flies were crossed to SM5/bwVI; TM3/TM6 stocks (see Lindsley and Zimm, 1992 for details of balancers) following standard procedures. All crosses were performed at $25^{\circ} \mathrm{C}$. For the South American and Australian cline a total of 80 and 90 female wild-type flies, respectively, from each population were crossed against balancers, and multiple flies were used in each generation to produce outbred chromosome substitution lines. Wing area was measured in flies reared at standard low density (50 larvae per vial, five vials per line). From each vial, 12 males and females were measured for all six lines from both continents.

\section{Inbred and parental lines for QTL mapping}

Isofemale lines were established from wild female flies collected at two sites from the east coast of Australia. Flies from the northern population near Cape Tribulation in Queensland (MEG, latitude: 16.85, longitude: 145.46) were collected in March 1998 and from the southern population at Domineys in Tasmania (DOM, 43.09, 147.24) in January 1998. These lines were maintained in the lab on standard fly food at $25^{\circ} \mathrm{C}$ for $10-14$ generations. Seven DOM and five MEG lines were inbred, first by mating two males with two females for five generations, followed by single pair matings for another five generations. Following inbreeding, lines were maintained at $25^{\circ} \mathrm{C}$ for another 16 generations until the start of the experiment.

Parental lines for generation of $\mathrm{F}_{3}$ flies were chosen according to the number of diagnostic maternal and paternal markers and their phenotypic difference in size. One fly each from all 12 inbred lines was genotyped for a subset of 19 microsatellite markers. Wing size was measured for 10 females from each line, reared in one vial per line at 50 first instar larvae per vial. Size differences between females of MEG and DOM lines ranged from 2.6 to 7.2 standard deviations. The inbred lines MEG4 and DOM20 gave the most optimal combination of size difference and number of diagnostic markers. To estimate possible effects of inbreeding depression on body size, wing size was compared between inbred lines and wild type isofemale lines. Changes in body size due to inbreeding only occurred in MEG4, which was significantly larger after inbreeding $(F=52.03, P=0.002)$. The overall size difference between MEG4 and DOM20 stayed highly significant $(F=345.87, P<0.001)$.

There was no significant difference in the number of cells in the measured compartment of the wing between 
females of the parental lines ( $t$-test: $P=0.47$ ). If cell size contributed to the difference in wing area, we would expect a significant difference in the number of trichomes counted in the sampling square. Therefore, essentially all the difference in total wing area was due to variation in cell number.

\section{Generation of $\mathrm{F}_{3}$}

To further increase the number of diagnostic markers within the chosen line combination, five crosses were set up with a MEG4 female and a DOM20 male. One cross produced no progeny. The parents of the remaining four crosses were genotyped for a set of 56 microsatellite markers suitable for mapping (list can be obtained from the authors; for further details on markers see http:// www.ucl.ac.uk/biology/goldstein/Mlist1.htm). Between 32 and 37 markers were homozygous and different between the parental flies and the cross with the highest number was chosen for further analysis. To minimize the influence of non-genetic maternal effects on body size, density was controlled for two generations prior to measurements. $\mathrm{F}_{2}$ flies were set up by counting 500 eggs into a bottle of standard fly medium. In the next generation, eggs were collected from the randomly intercrossed $\mathrm{F}_{2}$ flies on grape juice agar and 500 first instar $\mathrm{F}_{3}$ larvae were picked into one large bottle with standard fly medium to reduce environmental effects (eg, due to slight changes in food composition in different bottles). All flies were reared at $25^{\circ} \mathrm{C}$. Two weeks after the larval pick, all adult female flies were frozen for size measurement and scoring of microsatellite markers.

\section{Size measurement}

The right wing of female flies was mounted with the dorsal side facing up on microscope slides in Aquamount. Wing images were captured using a compound microscope ( $2.5 \times$ objective) with an attached video camera connected to a Macintosh computer. Wing area was determined using the Object-Image program (by Norbert Vischer, based on the public domain NIH Image and available at http://simon.bio.uva.nl) to calculate areas and record coordinates of all landmarks. The area measured consisted of a polygon whose vertices were the humeral-costal break, the distal ends of longitudinal veins, and the base of the alula. The polygon area measurement is highly correlated with wing area as measured by tracing an outline on a graphics tablet (Gilchrist and Partridge, 1999), and has the advantages of being faster and more reproducible. Sizes were recorded in pixels and converted into $\mathrm{mm}^{2}$.

\section{Molecular markers}

A total of 41 microsatellite markers was used to determine parental alleles in the $\mathrm{F}_{3}$ offspring. Markers were dispersed on all three chromosomes with 10 on the $X$ chromosome, 17 on the second and 14 on the third. The average spacing was $6.7 \mathrm{cM}$ on the $X$ chromosome, $6.6 \mathrm{cM}$ on the second and $7.9 \mathrm{cM}$ on the third chromosome. The largest gap of $28.5 \mathrm{cM}$ was found on the third chromosome. The sources of the markers are given in Appendix 1. Eight of the microsatellite markers used were identified using the BDGP Pattern Search (http://www.fruitfly.org). Primers were designed using the online software Primer3.cgi (version 0.2b). For three out of the 29 markers that were previously identified, either one or both primers were redesigned to optimize mulitplex conditions.

\section{Genotyping}

DNA was extracted from single $F_{3}$ flies (Gloor et al, 1993). PCR reactions were performed in pre-aliquoted plates (Advanced Biotechnologies) containing $0.2 \mathrm{mM}$ each dNTP, $2.5 \mathrm{mM} \quad \mathrm{MgCl}_{2}, \quad 75 \mathrm{mM}$ Tris-HCL, $20 \mathrm{mM}$ $\left(\mathrm{NH}_{4}\right)_{2} \mathrm{SO}_{4}, 0.01 \%$ Tween 20 and 0.31 units of Taq polymerase. One microlitre each of DNA extract and primermix of between two and five markers were added for a final reaction volume of $13 \mu \mathrm{l}$. Concentrations for each primer pair in the mix were adjusted and final concentrations in the PCR reactions ranged from $0.04-0.54 \mu \mathrm{M}$ (Appendix 1). Two markers (DRONANOS, AC004307) were amplified on their own. All amplifications were carried out in a GeneAmp PCR 9700 system (Perkin-Elmer) using the cycle profile: $94^{\circ} \mathrm{C} 4^{\prime},\left(94^{\circ} \mathrm{C} 30^{\prime \prime}, 53^{\circ} \mathrm{C} 30^{\prime \prime}, 72^{\circ} \mathrm{C}\right.$ $\left.30^{\prime \prime \prime}\right) \times 25,72^{\circ} \mathrm{C} 8^{\prime}$. For specificity and sensitivity of the PCR reaction, amplifications were prepared in the following order: DNA was pipetted into the lids of 96-well plates $(0.2 \mathrm{ml})$; primer-mix was added to the prealiquoted PCR reaction mix; lids were attached, the plate centrifuged $1^{\prime}$ at $1000 \mathrm{rpm}$ and placed in the preheated thermocycler ('hot start' PCR). Allele sizes were scored on an automated sequencer (ABI 377) by using GeneScan 2.1 and Genotyper 2.5 software (Perkin-Elmer). The following multiplex or primer combinations were pooled and run in the same gel lanes (Appendix 1): M8 and N5 (P1), M10 and marker 95 and 20 (P2), M13 and M14 (P3). Therefore, to score genotypes at 41 loci, 14 PCR reactions and 10 gel-lanes were needed for each individual.

\section{Analysis of chromosome substitution}

The contributions of the individual chromosomes and their interactions to variation in body size were analyzed separately for each sex and continent using a least squares analysis (Kearsey and Pooni, 1996). Data from flies from different vials were pooled, because no significant vial effects were found. The analysis was performed using the software package JMP 3.2.2 (SAS, 1997).

\section{QTL analysis}

QTL were identified by composite interval mapping (Zeng, 1994) using model 6 (Composite Interval Mapping) in QTL Cartographer 1.14 (Basten et al, 1997; $\mathrm{ftp}: /$ /statgen.ncsu.edu/pub/qtlcart/) on a randomly mated third generation intercross (RF3). CIM tests the hypothesis of the presence of a QTL in a given interval between two adjacent markers while simultaneously controlling for effects of linked QTL on the same chromosome outside the test interval and a predefined test window to the left and the right of the interval. This window size was chosen to be $10 \mathrm{cM}$ as default. Variation in window size $(1 \mathrm{cM}, 5 \mathrm{cM}$ and $15 \mathrm{cM})$ did not change the number of QTL identified by this procedure. Model 6 also controls for genetic background by including a number of markers, in this case nine, that were previously ranked in a forward regression with backward elimination procedure. The statistical properties of CIM mapping procedures have been analysed in detail by Zeng (1994). Briefly, CIM provides an interval test in which the test statistic is unaffected by all statistically relevant QTL which are located outside the interval being tested and two adjacent intervals. Therefore, its precision is increased due to an increased sensitivity of the test statistic to the position of a QTL. However, it is conditioned on other markers in the multiple regression analysis, 
which may entail a loss of statistical power to detect QTL (Zeng, 1993).

The likelihood ratio test statistic $L R=-2 \ln \left(L_{0} / L_{1}\right)$ is the ratio of the likelihood that a QTL is present in a test interval relative to the null hypothesis of no QTL in the interval. Following convention, the likelihood ratio test statistics were converted to LOD scores (Lander and Botstein, 1989). The test statistic is approximately $\chi^{2}$-distributed with the degrees of freedom given by the extra number of fitted parameters in the full model (Lynch and Walsh, 1998), and was evaluated every centimorgan. The experiment-wise significance level for each analysis was determined by 1000 permutations in which phenotypes are shuffled against genotypes and the analyses redone (Churchill and Doerge, 1994). In each permutation the highest test statistic was recorded, and these were ordered at the end of the permutations. The 95th percentile corresponds to an experimentwise significance level at $\alpha=0.05$. The data follow a normal distribution (K-S Test: $P=0.336)$.

Comparison of QTL and clinal microsatellite locations A previous survey of microsatellite allele frequency variation in natural populations along the latitudinal body size cline on the east coast of Australia revealed that, while most markers show a non-clinal pattern in the frequency of their most common allele (MCA), five loci showed a significant relationship between MCA frequency and latitude (Gockel et al, 2001). To test the coincidence of identified QTL locations with the locations of the five clinal microsatellite loci we performed two resampling tests. These tests were adapted from Macdonald and Goldstein (1999) who used them to compare QTL locations in different species. First, we divided the genome into 150 segments. We then summed the LOD scores of the segments that correspond to the position of the five clinal loci. To determine if this total was significantly greater than expected, we compared it with the summed LOD scores of five random segments in 10000 simulations. Second, to focus only on location, we summed distance of the clinal segments to the nearest significant QTL peak, and then compared it with the summed distance of five random segments in 10000 simulations. For both tests, a significant association between a QTL and clinal microsatellite locations was deemed to occur if the observed totals occurred outside of the upper or lower $95 \%$ confidence limits of the random distributions.

\section{Results}

\section{Chromosome substitution}

Wing areas of the chromosome substitution lines from each continent are shown in Figure 1. On average, the substitution of a big chromosome 3 in a small background (SSB) accounts for $77 \%$ of the size difference between big and small. Substituting big X or second chromosomes into a small background (BSS, SBS) each account for $13 \%$ of the overall difference in size between big and small. Results for a least squares analysis are shown in Table 1. In Australian and South American females all three chromosomes had a significant effect on body size, in males of both continents only chromosomes 2 and 3 . In all cases chromosome 3 showed the largest effects. Significant interactions were found between chro-
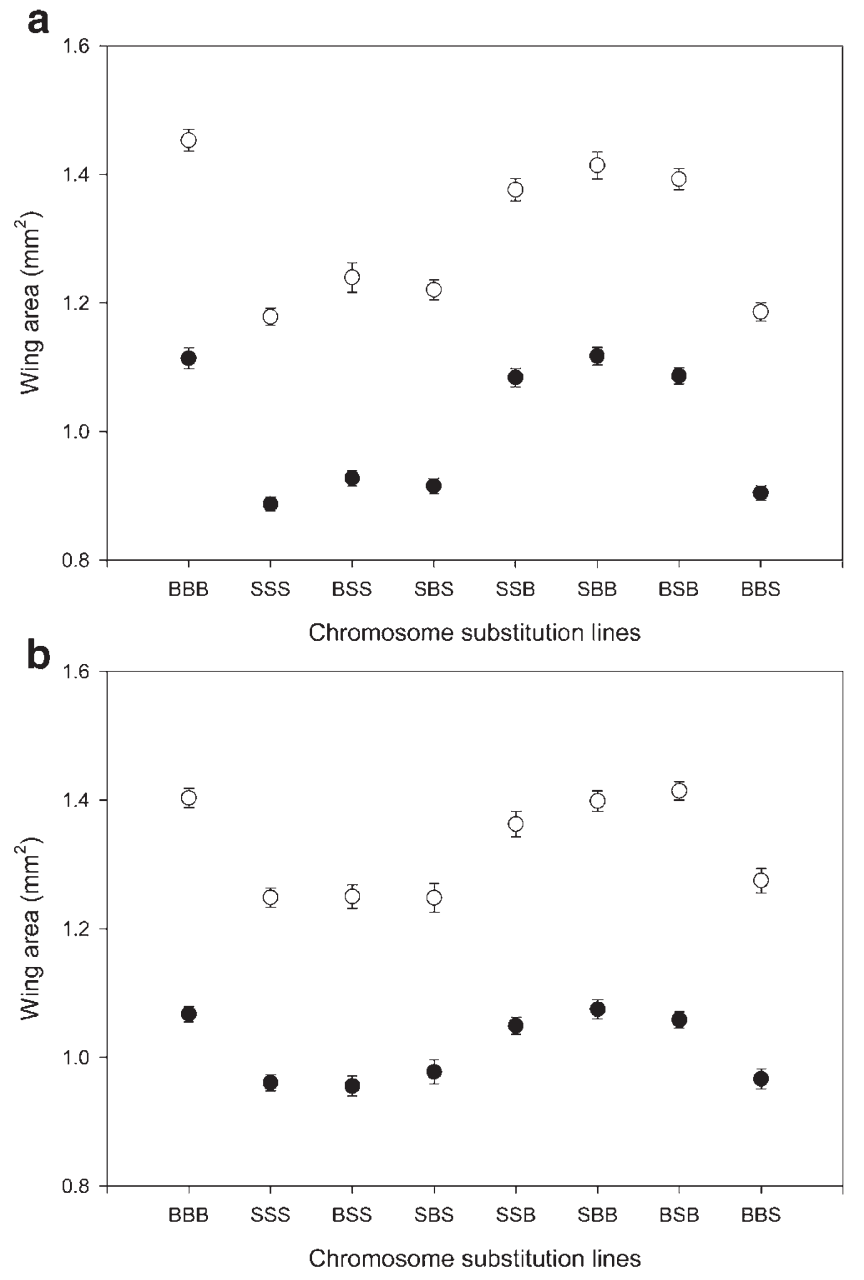

Figure 1 Wing areas of (a) South American and (b) Australian chromosome substitution lines. B represents big (high latitude) chromosome, S represents small (low latitude) chromosome. Position of the letter represents chromosome number, e.g. BBS = big chromosomes 1 and 2, small chromosome 3 (closed circles $=$ females, open circles $=$ males; error bars $=95 \%$ confidence intervals).

mosomes 2 and 3 and between all three chromosomes in the South American flies and between all three chromosomes in the Australian females. Australian males showed no significant interaction effects between chromosomes.

Phenotypic variation in the Australian $\mathrm{F}_{3}$ generation A total of 178 adult females were retrieved from the 500 first instar larvae. Wing area varied between 0.78 and $1.46 \mathrm{~mm}^{2}$ with a mean of $1.29 \mathrm{~mm}^{2}$ (SD 0.091). Although the sizes of $\mathrm{F}_{3}$ fly wings were intermediate between those of the parental lines, the results for these two generations are not directly comparable, because the measurements were taken at different times, and could therefore have been influenced by environmental factors such as minor variations in food composition.

During the process of mounting fly wings, the overall phenotype of each individual fly was also noted. A total of 16 flies showed a low level of pigmentation and were classified as 'runts'. Some of these flies also had a strikingly small body size. When wing area was measured, 
Table 1 Least squares analysis of chromosome substitution lines $(\mathrm{df}=1)$

\begin{tabular}{|c|c|c|}
\hline Source & Sums of Squares & $P$ \\
\hline \multicolumn{3}{|l|}{ South America, Female } \\
\hline X Chromosome & 0.047 & 0.001 \\
\hline Chromosome 2 & 0.052 & $<0.001$ \\
\hline Chromosome 3 & 4.588 & $<0.001$ \\
\hline X Chr $\times$ Chr 2 & 0.038 & 0.003 \\
\hline $\mathrm{X}$ Chr $\times$ Chr 3 & 0.006 & 0.245 \\
\hline Chr $2 \times$ Chr 3 & 0.085 & $<0.001$ \\
\hline X Chr $\times$ Chr $2 \times$ Chr 3 & 0.098 & $<0.001$ \\
\hline \multicolumn{3}{|l|}{ South America, Males } \\
\hline X Chromosome & 0.006 & 0.112 \\
\hline Chromosome 2 & 0.031 & $<0.001$ \\
\hline Chromosome 3 & 4.275 & $<0.001$ \\
\hline $\mathrm{X}$ Chr $\times$ Chr 2 & 0.024 & 0.002 \\
\hline $\mathrm{X}$ Chr $\times$ Chr 3 & 0.007 & 0.094 \\
\hline Chr $2 \times$ Chr 3 & 0.022 & 0.003 \\
\hline $\mathrm{X}$ Chr $\times$ Chr $2 \times$ Chr 3 & 0.015 & 0.014 \\
\hline \multicolumn{3}{|l|}{ Australia, Females } \\
\hline X Chromosome & 0.053 & $<0.001$ \\
\hline Chromosome 2 & 0.018 & 0.048 \\
\hline Chromosome 3 & 2.253 & $<0.001$ \\
\hline $\mathrm{X}$ Chr $\times$ Chr 2 & 0.003 & 0.384 \\
\hline X Chr $\times$ Chr 3 & 0.006 & 0.257 \\
\hline Chr $2 \times$ Chr 3 & 0 & 0.985 \\
\hline X Chr $\times$ Chr $2 \times$ Chr 3 & 0.038 & 0.004 \\
\hline \multicolumn{3}{|l|}{ Australia, Males } \\
\hline X Chromosome & 0.002 & 0.485 \\
\hline Chromosome 2 & 0.028 & 0.002 \\
\hline Chromosome 3 & 1.104 & $<0.001$ \\
\hline X Chr $\times$ Chr 2 & 0.004 & 0.275 \\
\hline $\mathrm{X}$ Chr $\times$ Chr 3 & 0.002 & 0.406 \\
\hline Chr $2 \times$ Chr 3 & 0 & 0.762 \\
\hline $\mathrm{XChr} \times \mathrm{Chr} 2 \times \operatorname{Chr} 3$ & 0.001 & 0.593 \\
\hline
\end{tabular}

four of these runts had extremely small wings and were identified as outliers. All analyses were carried out for the remaining 174 individuals which now showed average and minimum sizes of 1.30 (SD 0.077) and $1.04 \mathrm{~mm}^{2}$, respectively.

\section{QTL identified for wing area}

LOD values were calculated for wing area analysed by CIM (Figure 2). By using appropriate unlinked markers as cofactors, CIM partly accounts for the segregation variance generated by unlinked QTL, while the effects of linked QTL can be reduced by including markers linked to the interval of interest (Lynch and Walsh, 1998; Zeng, 1994).

Two major QTL areas were identified by CIM, one at the very distal end of chromosome 2 and one that spans the larger part of chromosome 3 to the right (Figure 2). The LOD scores ranged between 15.73 and 17.17. Several smaller peaks with LOD scores ranging from 3.42 to 5.17 were detected on chromosome 2 , and there was one on 3L. However, in many cases the valleys of these peaks did not fall below the significance value (3.1), so the actual number of QTL is unknown. No QTL were detected on the $\mathrm{X}$ chromosome. Reducing the density of markers on the 3rd chromosome, so they were more evenly spaced, did not improve the precision of the likelihood curve (data not shown).
QTL Cartographer (Basten et al, 1997) identified a total of 14 peaks, seven each on the 2 nd and 3 rd chromosome. Genetic and cytological peak locations and ranges are shown in Table 2. The peak ranges were defined as the area marked by the lowest LOD scores adjacent to either side of an identified peak.

The long flat LOD curve, especially on chromosome three, is indicative of a large genomic area with reduced recombination. In Drosophila, and other invertebrate species, natural inversions do occur which could lead to a similar pattern in the likelihood curve and therefore confound the results of the QTL analysis. In the lines analysed in this study, there is evidence of recombination events in 13 individuals between markers DMU43583 and DMU43090, indicating that this region is not a single large inversion. However, it cannot be excluded that it holds one or a few small inversions that ultimately lead to a reduced amount of recombination for the whole area.

Co-localization of QTL and clinal microsatellite locations Four out of five of the clinal microsatellite loci identified in Gockel et al (2001) fell within the range of a QTL peak. Two of these markers have also been used in this study (AC004579, DMTRXIII). MCA frequency at locus AC004579 (filled circle in Figure 3) significantly increased with latitude, but negative relationships with latitude were found for markers DMTRXIII, DMU25686 and AC008193, which are all located on the third chromosome. DMU25686 and AC008193 are in close proximity to each other (4 cM apart) and fall under the same peak range (peak 12; Table 2). DMTRXIII is further upstream and falls under the range of peak 10. AC004579 is located on the second chromosome and falls under the range of peak 3. With the exception of locus DMU14395, a trend can be seen towards clinal microsatellite markers that are located within genomic areas that contain QTL for a trait that shows clinal variation along the same gradient (bottom graphs in Figure 2). Despite this trend, however, resampling tests performed on both the summed LOD and summed distance to nearest QTL peak revealed that the locations of identified QTL and clinal microsatellite loci were not significantly associated. The summed LOD at the locations of the five clinal markers was 53.7, which was within the range of the $95 \%$ confidence intervals (CI) of the random distribution (summed LOD, 9.0 to 61.7). The summed distance to nearest QTL peak of the clinal markers was 5.0, which was also within the range of the 95\% CI of the random distribution (sum distances, 0 to 121).

\section{Discussion}

In this study we have identified chromosomal and QTL effects associated with phenotypic natural variation in body size in $D$. melanogaster. We further propose a simple method of how QTL for clinally varying traits could be identified in natural populations. This method implies that genetic markers like microsatellites that are linked to a QTL for the trait under clinal selection will also show clinal variation in their allele frequencies.

\section{Chromosome substitution}

Chromosome three had the largest effect on body size in both the Australian and the South American cline, accounting for the majority of the body size differences 

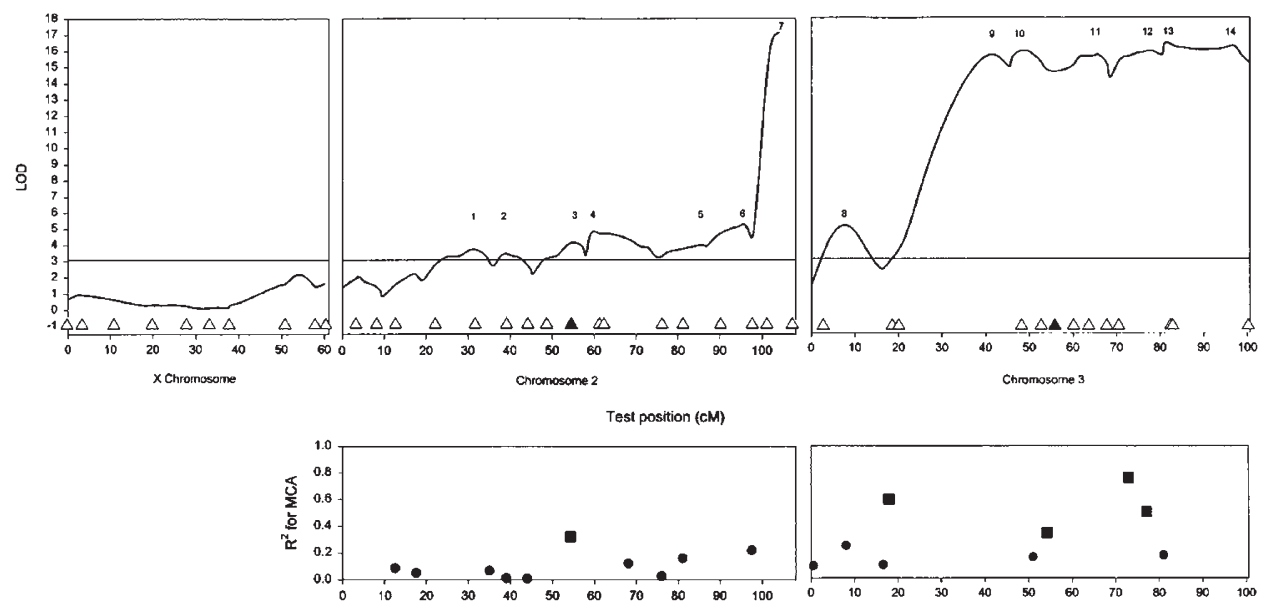

Genetic location

Figure 2 LOD profile for wing area based on CIM mapping and experimentwise critical value threshold (3.1) for wing area. Peak numbers are indicated for CIM as in Table 2. Triangles on the x-axis correspond to marker positions. Interval sizes correspond to map distances between markers in cM. Two markers (AC004579, DMTRXIII) that previously showed significant clinal variation along a latitudinal transect are indicated as black triangles. Underneath the LOD profiles are $\mathrm{R}^{2}$ values for the most common alleles (MCA) at 19 microsatellite loci regressed over latitude analyzed in a previous study (Gockel et al, 2001; loci located only on the second and third chromosome). Loci for which latitude explained a significant proportion of the variation are shown as squares.

Table 2 Locations and range of LOD peaks for body size identified with composite interval mapping (CIM)

\begin{tabular}{|c|c|c|c|c|c|c|}
\hline \multirow[t]{2}{*}{$\begin{array}{l}\text { Chromo- } \\
\text { some }\end{array}$} & \multirow[t]{2}{*}{ Peak } & \multicolumn{2}{|c|}{$\begin{array}{l}\text { Map position } \\
\text { (LOD peak) }\end{array}$} & \multicolumn{2}{|c|}{ Map position (range) } & \multirow[t]{2}{*}{$L O D$} \\
\hline & & $\begin{array}{l}\text { genetic } \\
(c M)\end{array}$ & $\begin{array}{l}\text { cyto- } \\
\text { logical }\end{array}$ & $\begin{array}{l}\text { genetic } \\
(c M)\end{array}$ & $\begin{array}{l}\text { cyto- } \\
\text { logical }\end{array}$ & \\
\hline 2 & 1 & 30.5 & $28 \mathrm{~F}$ & $28.5-34.5$ & $28 \mathrm{C}-29 \mathrm{~F}$ & 3.73 \\
\hline 2 & 2 & 38.0 & $30 \mathrm{~F}$ & 38-41 & 30F-31D & 3.42 \\
\hline 2 & 3 & 55.3 & 41D-F & $49.5-58$ & 34D-44D & 4.17 \\
\hline 2 & 4 & 61.1 & $45 \mathrm{~F}$ & 58-75 & $44 \mathrm{~A}-52 \mathrm{D}$ & 4.75 \\
\hline 2 & 5 & 86.0 & 55D-F & $75-87$ & 52B-56B & 4.03 \\
\hline 2 & 6 & 94.5 & 57D-E & 87-98 & $56 \mathrm{~A}-58 \mathrm{D}$ & 5.17 \\
\hline 2 & 7 & 104.0 & $59 \mathrm{~F}$ & 98-108 & 58B-59F & 17.17 \\
\hline 3 & 8 & 8.0 & 63D-F & 4-12 & $62 \mathrm{~F}-64 \mathrm{C}$ & 5.17 \\
\hline 3 & 9 & 41.5 & 70C-D & $19.5-45.5$ & $65 \mathrm{E}-75 \mathrm{D}$ & 15.73 \\
\hline 3 & 10 & 48.0 & $84 \mathrm{~A}$ & $45.5-55.7$ & 73D-88D & 15.95 \\
\hline 3 & 11 & 65.7 & 91E-F & $55.7-68.5$ & $88 \mathrm{C}-92 \mathrm{E}$ & 15.73 \\
\hline 3 & 12 & 78.5 & $94 \mathrm{E}-\mathrm{F}$ & $68.5-80.5$ & 92E-95B & 15.92 \\
\hline 3 & 13 & 81.0 & $95 \mathrm{C}$ & 80.5-91 & 95B-97D & 16.43 \\
\hline 3 & 14 & 97.0 & $98 \mathrm{E}-\mathrm{F}$ & 91-100.5 & 97B-99F & 16.26 \\
\hline
\end{tabular}

between cline ends (approximately 77\%). Whether this large effect is due to few genes with major effects or a large number of genes on the third chromosome with minor effects remains to be tested. The $X$ and second chromosome also had significant but much smaller effects on body size in both continents. The similarities in the chromosomal basis of size variation in the two clines suggest that a large part of the response to selection may be achieved in a similar way in the two clines examined.

In addition to the effects of individual chromosomes on body size, interactions between chromosomes also had an effect on size in the clines studied. Interactions occurred between $X$ and second chromosome in South America and between chromosomes two and three suggesting epistasis among loci on different chromosomes. The difference in interactions between the continents support

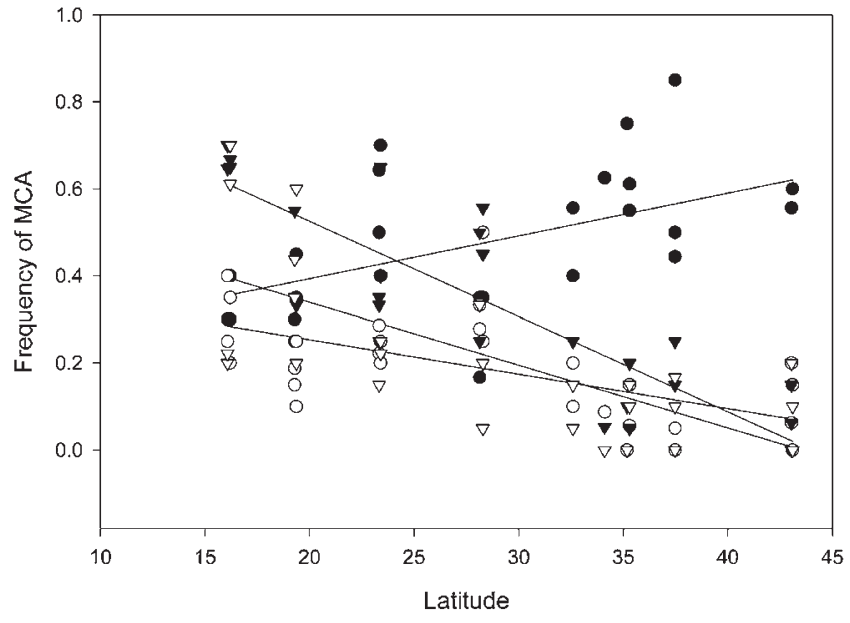

Figure 3 Frequencies of the most common allele and regression lines of markers AC004579 (filled circle), DMTRXIII (open circle), DMU25686 (filled triangle) and AC008193 (open triangle) in natural populations along a latitudinal transect in Australia. All of these markers have been identified to fall within a range under significant QTL peaks.

the results of a previous study which showed that there are differences in the epistatic component of body size between South America and Australia (Gilchrist and Partridge, 1999). Sex differences in chromosomal contribution to body size were also found. In contrast to females, male flies of both continents did not show significant $X$ chromosome effects, indicating that the $X$ chromosome played a greater role in producing latitudinal variation in female body size than it did in male body size in both continents.

\section{QTL for body size in $D$. melanogaster}

We analysed the Australian cline in more detail by crossing two inbred parental flies from the extreme ends of the cline and mapping QTL in the $\mathrm{F}_{3}$ generation using a 
microsatellite map. Again, major effects were found on the third chromosome but also on the distal end of chromosome 2. These results confirm the results of the chromosome substitution analysis.

However, in contrast to the chromosome substitution, no QTL have been identified for the $X$ chromosome. Despite the flat likelihood curves, QTL Cartographer (Basten et al, 1997) identified a total of 14 QTL peaks. Half of these peaks are located on the second chromosome and have minor effects. In most cases, the LOD curve between identified QTL peaks does not fall below the significance threshold. This effect is particularly obvious for peaks with large effect. It is therefore difficult to estimate how many QTL are falling exactly underneath each of these areas and to place confidence limits around localized QTL peaks. Unless a QTL is isolated, ie it is the only QTL in a particular interval and the flanking intervals lack QTL, methods like CIM cannot separate out the effects of multiple linked QTL (Whittaker et al, 1996). In particular, if an interval contains multiple QTL, their effects and positions cannot be estimated and the correct number of QTL cannot be inferred (McMillan and Robertson, 1974). An increased number of intervals using a fine scale map should present a solution to this problem by isolating detected QTL and thereby clearly separating individual loci.

In experimental designs with relatively low power, eg, due to a low sample size, but with a large number of QTL it is likely that not all QTL will be detected. In such a scenario, the contributions of detected QTL can be overestimated (Lynch and Walsh, 1998). In a simulation study Beavis (1994) showed that in such cases the distribution of observed effects are skewed, with a few loci having large estimated effects and the rest small to modest effects. It is therefore still possible that loci which contribute to phenotypic variation in body size are located on the $\mathrm{X}$ chromosome, as found in the chromosome substitution, but have not been detected in the mapping procedure due to the relatively low power of this analysis. Nevertheless, few of no QTL are often detected on the X chromosome in D. melanogaster, and may be a consequence of the lower overall number of genes per centimorgan on this chromosome (Noor et al, 2001). The 'small $X$ effect' we report is unlikely to result from the properties of the QTL mapping algorithms, because we obtained very similar results in our chromosome substitution experiments.

\section{Detecting QTL in natural populations}

This study, like many other QTL analyses, aimed at inferring the genetic basis of body size variation from statistical estimates of changes of mean and variance due to crossing of parental flies that show a large phenotypic difference. However, to obtain a full understanding of the genetic architecture of a quantitative trait will require more fine-scale mapping to identify individual QTL, reliable estimates of QTL effects, genetic interactions (epistasis) as well as genotype-environment interactions and identification of loci that affect naturally occurring variation within and between populations of a single species and among species. In a previous study on the genetic structure of $D$. melanogaster populations along a body size cline in Australia five microsatellite loci have been identified that showed a significant relationship with latitude (Gockel et al, 2001). Four of these loci fall within the range of QTL peaks identified in this study. Clinality of such markers could be due to linkage disequilibrium with a QTL that it is under clinal selection. It is likely that, depending on the pattern of linkage disequilibrium and strength of selection, a number to markers in close proximity of the QTL show clinality. Identification of a cluster of clinally varying markers in a sample of natural populations could therefore offer a way to detect QTL for the trait under clinal selection. Two clinal markers (DMU25686 and AC008193) fall under the same QTL peak range on the third chromosome and are therefore good candidates to be linked to one or more loci in that area that are under clinal selection. Generally, a trend can be seen in that markers with an increased degree of clinality, ie, an increased proportion of variation due to the environmental gradient or cline, fall under identified QTL peak areas. However, our analyses of co-localization between identified QTL and clinal markers revealed no significant association. It may be that a larger set of markers or higher resolution QTL maps are needed for a more reliable significance test. Certainly the broad QTL peaks identified in this study are likely to have reduced the possibility of a significant result here. Clearly, it remains to be seen whether a dense map of informative markers in populations that show clinal trait variation along an environmental gradient can be used to detect QTL in non-model species with an otherwise restricted array of molecular tools.

As pointed out previously, the demographic history of microsatellites is the most likely alternative explanation to a selection-dependent process of clinal variation of allele frequencies (Gockel et al, 2001). Therefore, information like colonization history and general population dynamics, if available, should also be taken into consideration for such association studies. Identifying clinal allele frequencies in natural populations with clinal trait variation can only be the first step in the analysis of QTL underlying such traits.

\section{Future studies}

The QTL peaks that have been identified here are not genetic loci but chromosome regions that contain one or more loci affecting body size. The size of the genomic region in which QTL are localized depends on the density of the markers and the scale of the experiment. Fine scale mapping should, as indicated above, provide a solution to some caveats of this study. It has to be noted however that very large numbers of recombination events are necessary to break up linked genomic regions to the level of genetic loci. Furthermore, a simulation study based on mouse body weight selection experiments showed that it is highly unlikely to obtain a significant correlation between two QTL studies if the variation in the trait is explained by large numbers of QTL and the experimental populations are not closely related (Keightley and Knott 1999). Future fine scale mapping projects should therefore include replicate datasets to account for the possibility of segregating QTL.

\section{Acknowledgements}

We thank the Natural Environment Research Council for financial support and two anonymous referees for helpful comments on the manuscript. 


\section{References}

Azevedo RBR, French V, Partridge L (1996). Thermal evolution of egg size in Drosophila melanogaster. Evolution 50: 2338-2345.

Basten CJ, Weir BS, Zeng ZB (1997). QTL Cartographer: A Reference Manual and Tutorial for QTL Mapping. Department of Statistics, North Carolina State University: Raleigh, NC.

Beavis WD (1994). The power and deceit of QTL experiments: lessons from comparative QTL studies. In: 49th Annual Corn and Sorghum Research Conference, American Seed Trade Association: Washington. D.C. pp 252-268.

Churchill GA, Doerge RW (1994). Empirical threshold values for quantitative trait mapping. Genetics 138: 963-971.

Colson I, Macdonald SJ, Goldstein DB (1999). Microsatellite markers for interspecific mapping of Drosophila simulans and $D$. sechellia. Mol Ecol 8: 1951-1955.

Gilchrist AS, Partridge L (1999). A comparison of the genetic basis of wing size divergence in three parallel body size clines of Drosophila melanogaster. Genetics 153: 1775-1787.

Gloor GB, Preston CR, Johnson-Schlitz DM, Nassif NA, Phillis RW, Benz WK, Robertson HM, Engels WR (1993). Type I repressors of P element mobility. Genetics 135: 81-95.

Gockel J, Kennington WJ, Hoffmann AA, Goldstein DB, Partridge L (2001). Non-clinality of molecular variation implicates selection in maintaining a morphological cline of Drosophila melanogaster. Genetics 158: 319-323.

Goldstein DB, Clark AG (1995). Microsatellite variation in North American populations of Drosophila melanogaster. Nucleic Acids Res 23: 3882-3886.

Gurganus MC, Nuzhdin SV, Leips JW, Mackay TF (1999). Highresolution mapping of quantitative trait loci for sternopleural bristle number in Drosophila melanogaster. Genetics 152: 15851604.

Harshman LG, Hoffmann AA (2000). Laboratory selection experiments using Drosophila: what do they really tell us? TREE 15: 32-36.

Huey RB, Gilchrist GW, Carlson ML, Berrigan D, Serra L (2000). Rapid evolution of a geographic cline in size in an introduced fly. Science 287: 308-309.

Imasheva AG, Bubli OA, Lazebny OE (1994). Variation in wing length in Eurasian natural populations of Drosophila melanogaster. Heredity 72: 508-514.

James AC, Azevedo RBR, Partridge L (1995). Cellular basis and developmental timing in a size cline of Drosophila melanogaster. Genetics 140: 659-666.

James AC, Azevedo RBR, Partridge L (1997). Genetic and environmental responses to temperature of Drosophila melanogaster from a latitudinal cline. Genetics 146: 881-890.

Kearsey MJ, Pooni JS (1996). The Genetical Analysis of Quantitative Traits. Chapman and Hall: London, UK

Keightley PD, Knott SA (1999). Testing the correspondence between map positions of quantitative trait loci. Gene Res 74 : 323-328.

Lander ES, Botstein D (1989). Mapping mendelian factors underlying quantitative traits using RFLP linkage maps. Genetics 121: $185-199$.

Laurie CC, Bridgham JT, Choudhary M (1991). Associations between DNA sequence variation and variation in expression of the Adh gene in natural populations of Drosophila melanogaster. Genetics 129: 489-499.

Leips J, Mackay TF (2000). Quantitative trait loci for life span in Drosophila melanogaster: interactions with genetic background and larval density. Genetics 155: 1773-1788.

Lindsley DL, Zimm GG (1992). Genome of Drosophila melanogaster. Academic Press: San Diego.

Liu J, Mercer JM, Stam LF, Gibson GC, Zeng ZB, Laurie CC (1996). Genetic analysis of a morphological shape difference in the male genitalia of Drosophila simulans and D. mauritiana. Genetics 142: 1129-1145.
Long AD, Mullaney SL, Reid LA, Fry JD, Langley CH, Mackay TF (1995). High resolution mapping of genetic factors affecting abdominal bristle number in Drosophila melanogaster. Genetics 139: 1273-1291.

Long AD, Lyman RF, Morgan AH, Langley CH, Mackay TF (2000). Both naturally occurring insertions of transposable elements and intermediate frequency polymorphisms at the achaete-scute complex are associated with variation in bristle number in Drosophila melanogaster. Genetics 154: 1255-1269.

Lyman RF, Mackay TF (1998). Candidate quantitative trait loci and naturally occurring phenotypic variation for bristle number in Drosophila melanogaster. The delta- hairless gene region. Genetics 149: 983-998.

Lyman RF, Lai C, MacKay TF (1999). Linkage disequilibrium mapping of molecular polymorphisms at the scabrous locus associated with naturally occurring variation in bristle number in Drosophila melanogaster. Genet Res 74: 303-311.

Lynch M, Walsh B (1998). Genetics and Analysis of Quantitative Traits. Sinauer Associates Inc: Sunderland, Massachusetts.

Macdonald SJ, Goldstein DB (1999). A quantitative genetic analysis of male sexual traits distinguishing the sibling species Drosophila simulans and D. sechellia. Genetics 153: 1683-1699.

Mackay TFC (2001). Quantitative trait loci in Drosophila. Nat Rev 2: $11-20$.

McMillan I, Robertson A (1974). The power of methods for the detection of major genes affecting quantiative characters. Heredity 3: 349-356.

Noor MAF, Cunningham AL, Larkin JC (2001). Consequences of recombination rate variation on quantitative trait locus mapping studies: simulations based on the Drosophila melanogaster genome. Genetics 159: 581-588.

Nuzhdin SV, Dilda CL, Mackay TF (1999). The genetic architecture of selection response. Inferences from fine-scale mapping of bristle number quantitative trait loci in Drosophila melanogaster. Genetics 153: 1317-1331.

SAS Institute (1997). JMP statistics for the Mackintosh. Version 3.2.2. SAS Institute Inc: Cary, NC.

Schug MD, Wetterstrand KA, Gaudette MS, Lim RH, Hutter CM, Aquadro CF (1988). The distribution and frequencies of microsatellite loci in Drosophila melanogaster. Mol Ecol 7: 57-70.

vant Land J, vanPutten $P$, Zwaan B, Kamping A, vanDelden $W$ (1999). Latitudinal variation in wild populations of Drosophila melanogaster: heritabilities and reaction norms. J Evol Biol 12: 222-232.

Vieira CE, Pasyukova G, Zeng ZB, Hackett JB, Lyman RF, Mackay TF (2000). Genotype-environment interaction for quantitative trait loci affecting life span in Drosophila melanogaster. Genetics 154: 213-227.

Weber K, Eisman R, Morey L, Patty A, Sparks J, Tausek M, Zeng ZB (1999). An analysis of polygenes affecting wing shape on chromosome 3 in Drosophila melanogaster. Genetics 153: 773786 .

Whittaker JC, Thompson R, Visscher PM (1996). On the mapping of QTL by regression of phenotypes on marker type. Heredity 77: 23-32.

Zeng ZB (1993). Theoretical basis of separation of multiple linked gene effects on mapping quantitative trait loci. Proc Natl Acad Sci USA 90: 10972-10976.

Zeng ZB (1994). Precision mapping of quantitative trait loci. Genetics 136: 1457-1468.

Zeng ZB, Liu J, Stam LF, Kao CH, Mercer JM, Laurie CC (2000). Genetic architecture of a morphological shape difference between two Drosophila species. Genetics 154: 299-310.

Zimmerman E, Palsson A, Gibson G (2000). Quantitative trait loci affecting components of wing shape in Drosophila melanogaster. Genetics 155: 671-683.

Zwaan BJ, Azevedo RBR, James AC, van't Land J, Partridge L (2000). Cellular basis of wing size variation in Drosophila melanogaster: a comparison of latitudinal clines on two continents. Heredity 84: 338-347. 
Appendix 1 Multiplex combinations, GenBank accession numbers (Acc. No), cytological and genetic locations, fluorescent dye labels (forward primer), melting temperatures ( $\mathrm{tm}$ ) of both primers and primer pair endconcentrations of 58 microsatellite loci. References are given, when microsatellites are taken from the literature. Primer sequence information can be found on http://www.ucl.ac.uk/biology/goldstein/Mlist1.htm. $\left(\mathrm{H}_{\exp }=1-\sigma \times_{i}^{2}\right.$, where $\mathrm{x}_{i}^{2}$ are allele frequencies)

\begin{tabular}{|c|c|c|c|c|c|c|c|c|c|c|c|c|c|c|}
\hline $\begin{array}{l}\text { Multiplex } \\
\text { name }\end{array}$ & Pool & $\begin{array}{l}\text { Locus } \\
\text { no. }\end{array}$ & Acc. No. & $\begin{array}{l}\text { Cytological } \\
\text { location }\end{array}$ & $\begin{array}{l}\text { Genetic } \\
\text { location }\end{array}$ & $\begin{array}{l}\text { Repeat } \\
\text { type }\end{array}$ & $\begin{array}{l}\text { Size } \\
\text { range } \\
(b p)\end{array}$ & $\begin{array}{l}\text { No. of } \\
\text { alleles }\end{array}$ & $\begin{array}{l}\text { Dye } \\
\text { label }\end{array}$ & $\begin{array}{l}t m \\
\text { forward } \\
\left({ }^{\circ} \mathrm{C}\right)\end{array}$ & $\begin{array}{l}\text { tm } \\
\text { back- } \\
\text { ward } \\
\left({ }^{\circ} \mathrm{C}\right)\end{array}$ & $\begin{array}{l}\text { Endconc. } \\
\text { in } \\
P C R \\
(\mu M)\end{array}$ & $H_{e x p}$ & $\operatorname{Ref}^{b}$ \\
\hline \multirow[t]{5}{*}{ M1 } & & 4 & AF047180 & 1B8 & $1-0$ & (AT) 16 & $254-276$ & 3 & Fam & 58.3 & 57 & 0.13 & 0.84 & Col99 \\
\hline & & 64 & DMU96440 & 7B3 & $1-20$ & (AG)14 & $259-265$ & 3 & Hex & 58.0 & 56.9 & 0.13 & 0.68 & ColUP \\
\hline & & 1 & DMC114E2 & 3D1-E8 & $1-3.5$ & (AT)17 & $319-366$ & 3 & Tet & 57.2 & 57.8 & 0.09 & 0.76 & ColUP \\
\hline & & 114 & AC011068 & $11 \mathrm{~A}-11 \mathrm{~A}$ & $1-38$ & (CA)11 & $217-222$ & 5 & Fam & 55.8 & 57.3 & 0.05 & $0.68+$ & - \\
\hline & & 116 & AC012373 & 17B-17C & $1-60.5$ & (CA)12 & $197-211$ & 7 & Tet & 57.2 & 56.5 & 0.13 & $0.84 \dagger$ & - \\
\hline \multirow{4}{*}{\multicolumn{2}{|c|}{ N1 }} & 3 & DMU56661 & 4F1-F2 & $1-11$ & $(\mathrm{AC}) 15$ & $268-294$ & 4 & Tet & 58.4 & 57.1 & 0.08 & 0.76 & Col99 \\
\hline & & 141 & DROSEV & 10A1-A2 & $1-33.4$ & $\begin{array}{c}\text { (TTG)9, } \\
\text { (TA)7 }\end{array}$ & $306-323$ & 5 & Hex & 57.6 & & 0.23 & 0.68 & SCH98 \\
\hline & & 5 & AC004114 & 8E3-E4 & $1-28$ & $(\mathrm{AC}) 13$ & $204-222$ & 3 & Tet & 57.9 & 58 & 0.08 & 0.51 & ColUP \\
\hline & & 65 & DMARIADNE & 16E2-17B1 & $1-58$ & (AC)14 & $120-140$ & 3 & Fam & 56.8 & 57.2 & 0.15 & 0.66 & Col99 \\
\hline \multirow{4}{*}{\multicolumn{2}{|c|}{ N2 }} & 132 & AC009392 & $23 \mathrm{~A}-23 \mathrm{E}$ & $2-8$ & $(\mathrm{GA}) 9$ & 185-195 & 5 & Fam & 57.1 & 58 & 0.10 & $0.69+$ & - \\
\hline & & 24 & AC004441 & $22 \mathrm{~A} 3$ & $2-3$ & (AT) 20 & $167-197$ & 5 & Hex & 52.0 & 56.8 & 0.15 & 0.85 & ColUP \\
\hline & & 122 & AC005115 & 32D2-32D4 & $2-44$ & (GT)12 & $176-193$ & 6 & Tet & 57.3 & 57.2 & 0.06 & $0.73+$ & - \\
\hline & & 67 & DMU12269 & 31A1-A3 & $2-39$ & $(\mathrm{AAC}) 7$ & $233-248$ & 6 & Fam & 57.2 & 57 & 0.18 & 0.78 & ColUP \\
\hline \multirow{4}{*}{\multicolumn{2}{|c|}{ M5 }} & 98 & AC004373 & 24F1-F2 & $2-12.5$ & (AT)15 & 177-191 & 4 & Fam & 56.9 & 56.8 & 0.19 & 0.77 & Col99 \\
\hline & & 21 & AC004759 & 38E1-E9 & $2-54.3$ & (CCT)11 & $224-242$ & 3 & Tet & 57.7 & 57.5 & 0.19 & 0.7 & Col99 \\
\hline & & 71 & AC004516 & 52D2-D15 & $2-76$ & $(\mathrm{AC}) 18$ & $259-283$ & 7 & Hex & 52.4 & 57.4 & 0.06 & 0.84 & Col99 \\
\hline & & 22 & AC004365 & 58A4-B1 & $2-97.5$ & (AT)17 & $209-221$ & 3 & Fam & 57.6 & 66 & 0.04 & 0.66 & Col99 \\
\hline \multirow{3}{*}{\multicolumn{2}{|c|}{ N3 }} & 68 & AC006302 & 34C4-D2 & $2-48.5$ & $(\mathrm{AAT}) 11$ & 229-251 & 5 & Fam & 55.7 & 57.4 & 0.13 & 0.71 & Col99 \\
\hline & & 108 & $\mathrm{AC} 005974^{\mathrm{a}}$ & 46E1-E9 & $2-62.1$ & $(\mathrm{CT}) 9$ & 187-195 & 3 & Fam & 57.9 & 57.5 & 0.08 & 0.72 & - \\
\hline & & 19 & AC004641 & 53D1-E2 & $2-81$ & $(\mathrm{AC}) 22$ & $218-256$ & 8 & Hex & 57.2 & 58 & 0.06 & 0.89 & Col99 \\
\hline \multirow[t]{3}{*}{ M8 } & P1 & 29 & AC003052 & 27A2-B2 & $2-22$ & (AT) 23 & $258-296$ & 7 & Tet & 56.0 & 57.4 & 0.15 & 0.82 & Col99 \\
\hline & & 36 & AC005555 & 29A1-C1 & $2-31.5$ & (TG)12 & $154-172$ & 3 & Tet & 51.9 & 54.4 & 0.05 & 0.61 & Col99 \\
\hline & & 27 & AC006472 & 45E1-46A2 & $2-61$ & (AG)16 & $274-302$ & 5 & Fam & 58.1 & 58.3 & 0.05 & 0.67 & Col99 \\
\hline \multirow[t]{4}{*}{ M9 } & & 73 & AC004343 & 62A1-A2 & $3-0.5$ & (TC) 12 & $219-231$ & 6 & Fam & 57.4 & 57.1 & 0.11 & 0.73 & Col99 \\
\hline & & 51 & AC004767 & 65A6-B2 & $3-16.5$ & (TA) 14 & $296-328$ & 6 & Hex & 52.8 & 57.9 & 0.28 & 0.8 & ColUP \\
\hline & & 55 & DMU14395 & 65D1-D3 & $3-18$ & (TC)12 & $281-303$ & 4 & Fam & 50.6 & 56.8 & 0.06 & 0.72 & Col99 \\
\hline & & 87 & DMU1951 & $95 \mathrm{C}$ & $3-81$ & (TA) 16 & 190-206 & 4 & Hex & 57.6 & 58 & 0.09 & 0.62 & G\&C95 \\
\hline \multirow[t]{2}{*}{ M10 } & P2 & 92 & DROPROSA & $86 \mathrm{E} 3$ & $3-51$ & $\begin{array}{l}\text { (CA)6, } \\
\text { (GA)12 }\end{array}$ & $111-125$ & 3 & Fam & 57.5 & 57.1 & 0.11 & 0.6 & Col99 \\
\hline & & 52 & DMTRXIII & $88 \mathrm{~B} 3$ & $3-54.2$ & (CT)23 & $306-354$ & 10 & Tet & 58.4 & 57.5 & 0.11 & 0.91 & Col99 \\
\hline \multirow[t]{2}{*}{ N5 } & P1 & 136 & DMU43583 & 83A3-A5 & $3-46.5$ & $(\mathrm{AT}) 22$ & $215-247$ & 6 & Fam & 60.4 & 59.6 & 0.27 & 0.76 & ColUP.2 \\
\hline & & 143 & DMTF125 & $95 \mathrm{C} 6-\mathrm{C} 8$ & $3-81.5$ & $(\mathrm{CAG}) 6$ & $278-298$ & 4 & Hex & 60.5 & 59.8 & 0.13 & 0.84 & Col99.2 \\
\hline \multirow[t]{6}{*}{ M12 } & & 6 & DMU18774 & $13 \mathrm{D}$ & $1-51$ & (AC) 12 & $182-196$ & 5 & Tet & 77.4 & 52.8 & 0.07 & 0.63 & Col99 \\
\hline & & 127 & AC007647 & $89 B-89 C$ & $3-58.5$ & (CA)12 & 192-202 & 4 & Fam & 57.6 & 58 & 0.07 & $0.35 t$ & - \\
\hline & & 128 & AC009394 & $90 \mathrm{E}-90 \mathrm{~F}$ & $3-62$ & (AT)15 & $231-239$ & 4 & Tet & 57.5 & 57 & 0.27 & $0.71+$ & - \\
\hline & & 129 & AC009347 & $93 \mathrm{~A}-93 \mathrm{~A}$ & $3-69$ & (AT) 15 & $218-236$ & 8 & Hex & 58.0 & 57 & 0.14 & $0.42 \dagger$ & - \\
\hline & P2 & 95 & DRONANOS & 91F13 & $3-66.2$ & (TA) 18 & $108-124$ & 3 & Tet & 56.7 & 57.6 & 0.54 & 0.77 & G\&C95 \\
\hline & P2 & 20 & AC004307 & 56D11-E6 & $2-90$ & (AT)14 & $242-267$ & 3 & Fam & 57.0 & 58 & 0.54 & 0.74 & ColUP \\
\hline \multirow[t]{2}{*}{ M13 } & P3 & $47 \ddagger$ & DS08011 & 59A1-B2 & $2-101$ & $(\mathrm{GT}) 8$ & $100-110$ & 2 & Fam & 62.6 & 55 & 0.27 & - & SCLUP \\
\hline & P3 & $75 \ddagger$ & DMU43090 & 99D6-D9 & $3-99$ & (CAG)8 & $180-189$ & 2 & Tet & 57.2 & 55.5 & 0.27 & - & Col99 \\
\hline \multirow[t]{2}{*}{ M14 } & P3 & $145 \ddagger$ & AE003466 & 60E8-E10 & $2-108$ & (TC)9 & $195-201$ & 2 & Hex & 58.4 & 58.2 & 0.27 & - & - \\
\hline & P3 & $146 \ddagger$ & AF221066 & 100F5 & $3-103$ & (TA) 16 & $192-200$ & 2 & Fam & 57.8 & 56.8 & 0.27 & - & - \\
\hline
\end{tabular}

a GenBank sequence reversed compared to our sequence. † Variability calculated from 8 D. melanogaster (for details see text). $\ddagger$ Marker only tested in flies of this study.

bReference abbreviations: Col99=Colson et al (1999); ColUP = I. Colson, unpublished; SCH98= modified from Schug et al 1998; G\& C95 = Goldstein and Clark (1995); ColUP.2 = modified from I Colson, unpublished; Col99.2 = modified from Colson et al (1999); SCLUP = C Schlotterer, unpublished. 